Freiburg, THEP-96/7

gr-qc/9605011

\title{
CONSISTENTLY IMPLEMENTING THE FIELDS SELF-ENERGY IN NEWTONIAN GRAVITY
}

\author{
Domenico Giulini* \\ Fakultt für Physik, Universität Freiburg \\ Hermann-Herder Strasse 3, D-79104 Freiburg, Germany
}

\begin{abstract}
We consider in a pedagogical fashion alterations to Newtonian gravity due to the postulate that all energy corresponds to active gravitational mass when applied to the self-energy of the gravitational field. We show why a simple addition of $\frac{1}{c^{2}}$ times the gravitational field energy to the matter density in Newton's field equation is inconsistent. A consistent prescription is shown and discussed. The connection to general relativity is pointed out.
\end{abstract}

\section{Introduction}

The issue addressed in this letter arises if one wishes to model the self-coupling of the gravitational field within Newtonian gravity. Simple non-linear alterations of Newton's field equation are often employed as simplified models for general relativity. The purpose of this letter is to show how this can be done and to point out certain flaws in the usually accepted prescription, as for example given in [1][2].

To be more precise, we recall that the Newtonian gravitational field, $\varphi$, and the density of (ponderable) matter, $\rho$, obey

$$
\Delta \varphi=4 \pi G \rho
$$

where $G$ is Newton's gravitational constant. The force per unit volume is given by

$$
\vec{f}=-\rho \vec{\nabla} \varphi
$$

* e-mail: giulini@sun2.ruf.uni-freiburg.de 
Together these equations imply that in order to build up a field $\varphi$ from $\varphi=0$ one has to invest the work

$$
A=-\frac{1}{8 \pi G} \int_{R^{3}}\|\vec{\nabla} \varphi\|^{2} d V .
$$

If we add the assumption that all energy acts as active gravitational mass, according to $E=m c^{2}$, and also think of the integrand in (1.3) as representing energy density, we might be tempted to consider the modified equation

$$
\Delta \varphi=4 \pi G\left(\rho-\frac{1}{8 \pi G c^{2}}\|\vec{\nabla} \varphi\|^{2}\right)
$$

with the aim to incorporate into Newtonian gravity the following

Principle (P). All energy acts as source for the gravitational field.

A field equation satisfying $\mathbf{P}$ must be non-linear. One might wonder whether (1.4) gives a Newtonian model that satisfies $\mathbf{P}$. If it were true that it shared this qualitative feature with general relativity one might profitably employ this single scalar equation to study certain qualitative features of general relativity in a mathematically simpler environment. In fact, (1.4) is often proposed in pedagogical discussions to precisely this end. For example, in [2][3] the authors suggest that some useful lessons concerning the energy-regulating power of the gravitational field can be learned from model theories of charged particles based on (1.4). In passing we remark that (1.4) can be written in a linear form by making the field-redefinition $\psi:=\exp \left(\varphi / 2 c^{2}\right)$ :

$$
\Delta \psi=\frac{2 \pi G}{c^{2}} \rho \psi
$$

where the boundary conditions $\varphi(r \rightarrow \infty)=0$ translate to $\psi(r \rightarrow \infty)=1$. In the following we shall for simplicity always assume $\rho$ to have compact support $B \subset R^{3}$.

In section 3 we discuss what is wrong with a theory based on $(1.2,4)$ and suggest a different and consistent theory in section 4. Section 5 briefly discusses some properties of spherically symmetric solutions to the latter and section 6 points out the relation to general relativity. Section 2 summarizes some facts from Newtonian gravity. We employ the standard summation convention for repeated indices in up-down positions and use the euclidean metric $\delta_{a b}$ to raise and lower indices. $\nabla_{a}$ denotes the partial derivative with respect to $x^{a}$. 3-component vectors are also written with an arrow, $\vec{\xi}$, with $\vec{\xi} \cdot \vec{\eta}=\xi_{a} \eta^{a}$ denoting the scalar product. 


\section{Newtonian Recollections}

To see what is wrong with (1.4) it is helpful to first give a derivation of (1.3). Consider a one-parameter family of diffeomorphisms $s \mapsto \sigma_{s}$ such that $\sigma_{s=0}=i d$ and $\left.\frac{d}{d s}\right|_{s=0} \sigma_{s}(\vec{x})=\vec{\xi}(\vec{x})$. We wish to use $\sigma_{s}$ to redistribute the matter by dragging it along this flow. Pulling back the 3 -form $\rho d V$ by the inverse diffeomorphisms we obtain $\rho_{s} d V:=\left(\sigma_{s}^{-1}\right)^{*}(\rho d V)$ and hence for the Lie-derivative of the density $\rho$ along $\vec{\xi}$

$$
\delta \rho:=\left.\frac{d \rho_{s}}{d s}\right|_{s=0}=-\vec{\nabla} \cdot(\rho \vec{\xi})
$$

where here and in the following we use the variational symbol, $\delta$, for the derivative at $s=0$ and call it 'the variation' of the quantity in question.

The variation of the work done to the system is easily determined using (1.2):

$$
\delta A=-\int_{R^{3}} \vec{\xi} \cdot \vec{f} d V=-\int_{B} \varphi \vec{\nabla} \cdot(\rho \vec{\xi}) d V
$$

Equations $(2.1,2)$ imply

$$
\delta A=\int_{B} \varphi \delta \rho d V
$$

This equation is independent of the field equation. If we assume the validity of (1.1) throughout the (adiabatic) motion we can use it to eliminate $\delta \rho$ and write (2.3) solely in terms of $\varphi$. The result (1.3) then easily follows.

From $(1.1,2)$ it follows that the force per unit mass may be derived from a symmetric stress tensor, $f_{a}=-\nabla^{b} t_{a b}$, where

$$
t_{a b}=\frac{1}{4 \pi G}\left(\left(\nabla_{a} \varphi\right)\left(\nabla_{b} \varphi\right)-\frac{1}{2} \delta_{a b}\|\vec{\nabla} \varphi\|^{2}\right)
$$

so that

$$
\delta A=-\int_{R^{3}} f_{a} \xi^{a} d V=\int_{R^{3}} \xi^{a} \nabla^{b} t_{a b} d V=\int_{R^{3}} \nabla^{(a} \xi^{b)} t_{a b} d V .
$$

Here we assumed $\|\vec{\xi}(r \rightarrow \infty)\|<$ ar for some real constant $a$ and that derivatives of $\varphi$ fall off as fast as $r^{-2}$. Vector fields which satisfy $\nabla^{(a} \xi^{b)}=0$ (Killing equation) generate rigid motions and are given by $\vec{\xi}(\vec{x})=\vec{k}$ (translations) and $\vec{\xi}(\vec{x})=\vec{k} \times \vec{x}$ (rotations), for constant $\vec{k}$. For those $\delta A=0$, as it must be by the principle of action $=$ reaction. Otherwise the system would self-accelerate. 
Finally we define the gravitational mass as the total flux of the gravitational field $\vec{\varphi}$ out to infinity:

$$
M_{g}:=\lim _{r \rightarrow \infty} \frac{1}{4 \pi G} \int_{S_{r}^{2}} \vec{n} \cdot \vec{\nabla} \varphi d o
$$

$S_{r}^{2}$ denotes a two-sphere of radius $r, \vec{n}$ its (outward pointing) normal, and $d o$ the surface element on $S_{r}^{2}$. The limit of integrals in (1.14) is sometimes abbreviated by $\int_{S_{\infty}^{2}} \cdot$

\section{Why Inconsistent?}

Since in Newtonian theory $M_{g}=M_{m}:=\int \rho d V, M_{g}$ only depends on the amount but not on the distribution of matter and clearly $\mathbf{P}$ cannot be satisfied. Now, replacing (1.1) by (1.4), one obtains the following formula for the variation $\delta M_{g}$

$$
\delta M_{g}=\int_{B} \sum_{n=0}^{N-1} \frac{1}{n !}\left(\frac{\varphi}{c^{2}}\right)^{n} \delta \rho d V+\frac{1}{N ! c^{2 N}} \frac{1}{4 \pi G} \int_{R^{3}} \varphi^{N} \delta(\Delta \varphi) d V
$$

where we have used (1.4) $N$ times to replace $\Delta \varphi$. For a regular matter distribution $\varphi$ will be bounded, say $\varphi(\vec{x})<K, \forall \vec{x} \in R^{3}$. Also, the integral over $\frac{1}{4 \pi G} \delta(\Delta \varphi)$ just represents $\delta M_{g}$ so that the last term on the right hand side is majorized by $\frac{1}{N !}\left(K / c^{2}\right)^{N} \delta M_{g}$. It vanishes in the limit $N \rightarrow \infty$. In this limit the sum on the right side is just the exponential function. Thus we obtain the result:

$$
\delta M_{g}=\int_{B} \delta \rho \exp \left(\varphi / c^{2}\right) d V
$$

which, recalling (2.3), deviates from $\delta A / c^{2}$ by all the higher-than-linear terms in the expansion of the exponential. Hence $(1.2,4)$ violates $\mathbf{P}$. This is not really surprising, since (1.3) was derived under the assumption of $(1.1,2)$. Changing it to $(1.2,4)$ also invalidates (1.3). A correct procedure must iterate the step that led from (1.1) to (1.4). For example, the next (second) step would be to determine a modified expression for the gravitational field energy from $(1.2,4)$ and then change $(1.4)$ accordingly. Eventually this procedure should converge to a self-consistent field equation. However, as we will see in the next section, such a self-consistent field equation can actually be guessed directly. 
At the end of this section we also point out another flaw in the combination $(1.2,4)$. Using $(1.5)$ to replace $\rho$ in $(1.2)$ one easily derives

$$
f_{a}=-\exp \left(-\varphi / c^{2}\right) \nabla^{b}\left(\exp \left(\varphi / c^{2}\right) t_{a b}\right)
$$

with $t_{a b}$ from (2.4). From this expression it follows that the force density is not the divergence of a stress tensor. There are many ways to isolate the part that obstructs the right hand side of (2.11) to be written as the divergence of a symmetric tensor. Two obvious ways are

$$
\begin{aligned}
f_{a} & =-\nabla^{b} t_{a b}-\frac{1}{8 \pi G c^{2}}\|\vec{\nabla} \varphi\|^{2} \nabla_{a} \varphi \\
& =-\nabla^{b}\left[\left(1+\varphi / c^{2}\right) t_{a b}\right]+\frac{1}{8 \pi G c^{2}} \Delta \varphi \nabla_{a} \varphi^{2} .
\end{aligned}
$$

The system $(1.2,4)$ thus potentially violates the principle action $=$ reaction. $^{1}$

\section{A Consistent Modification}

Equation (3.2) was derived assuming (1.4) but not (1.2). If we maintain $(1.4,5)$ but call $\phi=c^{2} \exp \left(\varphi / c^{2}\right)$ rather than $\varphi$ the gravitational potential, we have $(3.2)$ just expressing the validity of $\mathbf{P}$, i.e. $c^{2} \delta M_{g}=\delta A$ with $\delta A$ given by (2.3). This re-interpretation implies that (1.2) has to be replaced by

$$
\vec{f}=-\rho \vec{\nabla} \phi
$$

and that $(1.4,5)$ written in terms of $\varphi$ reads

$$
\Delta \phi=\frac{4 \pi G}{c^{2}}\left(\rho \phi+\frac{c^{2}}{8 \pi G} \frac{\|\vec{\nabla} \phi\|^{2}}{\phi}\right) .
$$

To be sure, for explicit calculations one would preferably use (1.5) where $\psi=$ $c \sqrt{\phi}$. $\phi$ must satisfy the boundary conditions $\phi(r \rightarrow \infty)=c^{2}$. The Newtonian approximation is obtained from expanding $\phi=c^{2}+\varphi+O\left(\varphi^{2}\right)$ and keeping only linear terms in (4.2). Note that in the expression (2.6) for $M_{g}$ we must write $\phi$

\footnotetext{
1 To manifestly show a violation one should prove existence of a regular solution to (1.4) with $\varphi(r \rightarrow \infty)=0$ for which $\int f_{a} \xi^{a} \neq 0$ for some generator $\vec{\xi}$ of a rigid motion.
} 
instead of $\varphi$. But for $r \rightarrow \infty$ only the linear term in $\varphi$ contributes to the surface integral so that (3.2) is still valid. This is why (3.2) indeed expresses the validity of $\mathbf{P}$ for $(4.1,2)$. To be sure, once $(4.1,2)$ are established, the equation $c^{2} \delta M_{g}=\delta A$ is most easily proven directly. For completeness we give a short direct proof in the appendix. The point of our derivation of (3.2) was that it suggested the definition of $\phi$ in terms of $\varphi$ and hence (4.2). It is interesting to note that (4.2) is precisely the equation that Einstein already proposed before the advent of general relativity in 1912 [4].

Equations $(4.1,2)$ also manifestly implie the principle action $=$ reaction in the sense above. Indeed, we now have $f_{a}=-\rho \exp \left(\varphi / c^{2}\right) \nabla_{a} \varphi$. Replacing $-\rho \nabla_{a} \varphi$ by the right hand side of (3.3) just cancels the exponential function outside the derivative so that the remaining divergence can be rewritten in terms of $\phi$. This leads to the desired formula, $f_{a}=-\nabla^{b} t_{a b}$, with

$$
t_{a b}=\frac{1}{4 \pi G c^{2}}\left\{\frac{1}{\phi}\left[\left(\nabla_{a} \phi\right)\left(\nabla_{b} \phi\right)-\frac{1}{2} \delta_{a b}\|\vec{\nabla} \phi\|^{2}\right]\right\} .
$$

We may interpret the two terms on the right hand side of (4.2) as energy densities due to ponderable matter and the gravitational field respectively. The sum of both determines the convergence $\Delta \phi$ of the gravitational field $-\vec{\nabla} \phi$. Both terms are positive since $\phi$ is positive. This is in contrast to (1.4), where the Newtonian gravitational field energy was negative definite, which is usually said to have its origin in the attractivity of gravity. But of course here gravity is also attractive. What is different here is that the (rest-) energy of matter depends on the value of the gravitational potential at its location. This allows that a contraction of a matter distribution enhances the field energy although the total energy decreases. This is achieved by displacing the matter into regions of smaller gravitational potential and thereby sufficiently decreasing the matter part of the energy.

The total gravitational energy is given by

$$
E_{\text {total }}:=c^{2} M_{g}=\int_{B} \rho \phi d V+\frac{c^{2}}{8 \pi G} \int_{R^{3}} \frac{\|\vec{\nabla} \phi\|^{2}}{\phi} d V=: E_{\text {matter }}+E_{\text {field }}
$$

where the expression for $E_{\text {field }}$ can also be written in terms of an integral over $B$ (the support of $\rho$ ) only. To see this we recall that for large distances from the source 
we have the expansions for $\phi$ and $\psi$

$$
\begin{gathered}
\frac{\phi}{c^{2}}=1-\frac{G M_{g}}{c^{2} r}+O\left(r^{-2}\right) \\
\psi=1-\frac{G M_{g}}{2 c^{2} r}+O\left(r^{-2}\right)
\end{gathered}
$$

so that $E_{\text {total }}$ can also be expressed as an integral of $\frac{c^{4}}{2 \pi G} \Delta \psi=c^{2} \rho \psi$ (using (1.5)) over $B$. Replacing $E_{\text {total }}$ by this expression in $E_{\text {field }}=E_{\text {total }}-E_{\text {matter one obtains }}$

$$
E_{\text {field }}=c^{2} \int_{B} \rho \sqrt{\frac{\phi}{c^{2}}}\left(1-\sqrt{\frac{\phi}{c^{2}}}\right) d V
$$

\section{Solution for Homogeneous Spherical Star}

In this section we determine the gravitational field for the externally prescribed mass distribution

$$
\rho= \begin{cases}\frac{3 M_{m}}{4 \pi R^{3}} & \text { for } r<R \\ 0 & \text { for } r \geq R\end{cases}
$$

where $M_{m}$ is the total ('bare') mass of matter: $M_{m}=\int_{B} \rho d V$. It will be convenient to introduce the 'matter radius' $\mathcal{R}_{m}$ and the 'gravitational radius' $\mathcal{R}_{g}$ :

$$
\mathcal{R}_{m}=\frac{G M_{m}}{c^{2}}, \quad \mathcal{R}_{g}=\frac{G M_{g}}{c^{2}}
$$

and the abbreviation

$$
\omega=\sqrt{\frac{3 \mathcal{R}_{m}}{2 R}} \frac{1}{R} .
$$

We use (1.5), set $\psi(r)=\chi(r) / r$, and obtain

$$
\chi^{\prime \prime}= \begin{cases}\omega^{2} \chi & \text { for } r<R \\ 0 & \text { for } r \geq R .\end{cases}
$$

The general solution which makes $\phi$ (and hence $\psi$ ) finite at $r=0$ is

$$
\psi(r)= \begin{cases}K \frac{\sinh (\omega r)}{\mathcal{R}_{g}^{r}} & \text { for } r<R \\ 1-\frac{\mathcal{R}_{g}}{2 r} & \text { for } r \geq R .\end{cases}
$$


The integration constants $K$ and $\mathcal{R}_{g}$ are determined by the requirement that $\phi$ (and hence $\psi$ ) should be continuously differentiable at $r=R$ :

$$
\begin{aligned}
\mathcal{R}_{g} & =2 R\left[1-\frac{\tanh (\omega R)}{\omega R}\right] \\
K & =\frac{1}{\omega \cosh (\omega R)} .
\end{aligned}
$$

Fixing the radius $R$ in (4.6) gives us $\mathcal{R}_{g}$ as function of $\mathcal{R}_{m}$, i.e., the gravitational mass as function of the bare mass. In terms of the dimensionless quantities $y=$ $\mathcal{R}_{g} / R$ and $x=\mathcal{R}_{m} / R$ this reads:

$$
y=f(x)=2\left[1-\frac{\tanh (3 x / 2)^{\frac{1}{2}}}{(3 x / 2)^{\frac{1}{2}}}\right]
$$

which for $x \geq 0$ maps monotonically $[0, \infty] \rightarrow[0,2]$. For small $x$ one has $f(x)=$ $x-\frac{3}{5} x^{2}+\frac{51}{140} x^{3}+O\left(x^{4}\right)$. The fact that $f(x)<2 \quad \forall x \in R_{+}$means that the gravitational mass is bounded by a quantity depending only on the geometry (here $R$ ) of the mass distribution:

$$
M_{g}<R \frac{2 c^{2}}{G} .
$$

Note that this is achieved with all contributions to the gravitational mass on the right hand side of (3.3) being positive. No subtractions are taking place. Rather, high matter densities $\rho$ are suppressed by the small potentials $\phi$ produced by them (i.e. 'red-shifted' in general relativistic terminology). This can be seen in detail from the following expressions:

$$
\begin{aligned}
E_{\text {total }} & =\frac{2 R c^{4}}{G}\left[1-\frac{\tanh (\omega R)}{\omega R}\right] \\
& =M_{m} c^{2}\left[1-\frac{3 \mathcal{R}_{m}}{5 R}+O\left(\mathcal{R}_{m}^{2} / R^{2}\right)\right] \\
E_{\text {matter }} & =\frac{R c^{4}}{G}\left[\frac{\tanh (\omega R)}{\omega R}+\tanh ^{2}(\omega R)-1\right] \\
& =M_{m} c^{2}\left[1-\frac{6 \mathcal{R}_{m}}{5 R}+O\left(\mathcal{R}_{m}^{2} / R^{2}\right)\right] \\
E_{\text {field }} & =\frac{3 R c^{4}}{G}\left[1-\frac{\tanh (\omega R)}{\omega R}-\frac{1}{3} \tanh ^{2}(\omega R)\right] \\
& =M_{m} c^{2}\left[\frac{3 \mathcal{R}_{m}}{5 R}+O\left(\mathcal{R}_{m}^{2} / R^{2}\right)\right]
\end{aligned}
$$


where the second expressions on the right hand sides are expansions of the first in terms of $\mathcal{R}_{m} / R$. Also, recall that $\mathcal{R}_{m} c^{4} / G=M_{m} c^{2}$. Note the familiar $\frac{5}{3}$-term in (5.10b) for the Newtonian binding energy.

Decreasing $R$ for fixed $\mathcal{R}_{m}$ we see from (5.10b-12b) that to first approximation this enhances the field energy and at the same time decreases the matter energy twice as fast, so as to decrease the total energy by the same amount by which the field energy increased. Clearly the total energy must decrease in accordance with the attractivity of the gravitational interaction.

Coming back to (5.9) we next show that it remains valid for any spherically symmetric matter distribution. In particular, it remains valid for more realistic matter distributions (of compact support $r<R$ ) which are determined by a coupled system of (4.2) with some equations of state for the matter. The proof is simply this: For $r \geq R(5.4)$ is solved by $\chi_{+}(r)=r-\mathcal{R}_{g} / 2$ and by some function $\chi_{-}(r)$ for $r \leq R$. Continuity and differentiability of $\phi$ at $r=R$ is equivalent to

$$
\begin{aligned}
& \chi_{-}(R)=R-\frac{1}{2} \mathcal{R}_{g} \\
& \chi_{-}^{\prime}(R)=1 .
\end{aligned}
$$

Suppose $\chi(R) \leq 0$, then $\chi^{\prime \prime}=\frac{2 \pi G}{c^{2}} \rho \chi$ with $\rho \geq 0$ implies $\chi^{\prime \prime}(r) \leq 0$ for all $r \leq R$, with strict inequality if $r$ lies in the support of $\rho$. Equations $(5.13,14)$ now imply that the curve $r \rightarrow \chi_{-}(r)$ lies below the curve $r \rightarrow r-\frac{1}{2} \mathcal{R}_{g}$ for $r \leq R$, which in turn implies $\chi(r=0)<-\frac{1}{2} \mathcal{R}_{g}<0$, where the last inequality just expresses the positivity of the gravitational mass. But this contradicts the regularity of the gravitational potential which requires a finite value of $\psi(r=0)$ and thus $\chi(r=0)=0$. Hence we must have $\chi(R)>0$ or, by $(5.13), \mathcal{R}_{g}<2 R$.

Finally we mention that the finite (negative) bare mass for the point-charge model of ref. [V] crucially depends on taking $\varphi$ (and (1.4)) rather than $\phi$ as gravitational potential. In the latter case it unfortunately turns out to be infinite. At this state of affairs the finite bare mass obtained in $[\mathrm{V}]$ is not a consequence of $\mathbf{P}$, but rather an artifact of (1.4), which violates $\mathbf{P}$. But $\mathbf{P}$ seems crucial for any model of general relativity and hence (1.4) does not seem suited to model general relativity for questions concerning the energy regulating power of the gravitational field. 


\section{Connection with General Relativity}

Let us consider a Lorentz metric in which only the time-time component of the metric differs from its Minkowski value. We write

$$
d s^{2}=-2 \phi d t^{2}+d \vec{x} \cdot d \vec{x}
$$

and require that for large spatial distances this approaches the Minkowski metric:

$$
\lim _{\|\vec{x}\| \rightarrow \infty} \phi(\vec{x})=\frac{1}{2} c^{2}
$$

Using the parameter $t$, the equations for a timelike geodesic curve boil down to $(\dot{\vec{x}}=d \vec{x} / d t)$

$$
\ddot{\vec{x}}=-\vec{\nabla} \phi+\phi^{-1}(\dot{\vec{x}} \cdot \vec{\nabla} \phi) \dot{\vec{x}}
$$

which, neglecting terms $\propto(v / c)^{2}$ for the moment, are just the Newtonian equations of motion for a point mass in the external potential $\phi$. Setting for the moment $2 \phi=\psi^{2}$, the components of the Ricci tensor are most easily calculated with respect to the orthonormal tetrad: $e_{\hat{t}}=\psi^{-1} \partial_{t}, e_{\hat{a}}=\partial_{a}$ for $a=1,2,3$ (the hat over the indices signifies the orthonormality). We obtain:

$$
\begin{aligned}
R_{\hat{a} \hat{c}} & =-\psi^{-1} \psi_{, a, c} \\
R_{\hat{t} \hat{t}} & =\psi^{-1} \Delta \psi
\end{aligned}
$$

where $\Delta$ is just the ordinary Laplacian $\partial^{a} \partial_{a}$ and $\partial_{a} \psi=\psi_{, a}$. The scalar curvature then follows (summation over $\hat{a}$ ):

$$
R=R_{\hat{a} \hat{a}}-R_{\hat{t} \hat{t}}=-2 \psi^{-1} \Delta \psi
$$

Let us now consider Einstein's equations

$$
R_{\mu \nu}=\frac{8 \pi G}{c^{4}}\left(T_{\mu \nu}-\frac{1}{2} g_{\mu \nu} T_{\lambda}^{\lambda}\right)
$$

with an energy momentum tensor $T_{\mu \nu}=\rho u_{\mu} u_{\nu}$ for stationary pressureless dust of rest-mass-density $\rho$ and 4 -velocity $u=c \psi^{-1} \partial_{t}$. If we varied the Einstein-Hilbert 
action only within the class of metrics of the form (6.1) we would obtain only the $\hat{t} \hat{t}$-component of (6.7), which explicitly reads: $\Delta \psi=\frac{4 \pi G}{c^{2}} \rho \psi$. In terms of $\phi$ we have

$$
\Delta \phi=\frac{8 \pi G}{c^{2}}\left(\rho \phi+\frac{c^{2}}{16 \pi G} \frac{\|\vec{\nabla} \phi\|^{2}}{\phi}\right) .
$$

This is almost (4.3) except for an additional factor of two in the $G$-dependence. But note that the boundary condition (6.2) differs from (4.5a) by a factor $\frac{1}{2}$ which implies that (6.8) and (4.2) have the same Newtonian limit. A solution of (4.2), like (5.5), can be easily turned into a solution to (5.6) if we multiply it by $\frac{1}{2}$ and replace $G$ by $2 G$. Thus we conclude that (3.3) is essentially the time-time-component of Einstein's equations. Note however that we cannot solve the full set of Einstein's equations with the ansatz (5.1). In fact, adding the trace of the spatial part to the $\hat{t} \hat{t}$-part, the left hand side is zero according to $(5.4,5)$ whereas the right side is easily seen to be proportional to $T_{\hat{t} \hat{t}}$. So there is no solution, at least if the matter satisfies the dominant energy condition $\left|T_{\hat{t} \hat{t}}\right| \geq\left|T_{\hat{a} \hat{c}}\right|$. Note that the trace of Einstein's equations (5.7), $R=-\frac{8 \pi G}{c^{4}} T$, is already in contradiction to their $\hat{t} \hat{t}$ component, since $T=T_{\hat{a} \hat{a}}-T_{\hat{t} \hat{t}}=-T_{\hat{t} \hat{t}}=-\rho c^{2}$ so that with (5.6) the trace part reads $\Delta \psi=-\frac{4 \pi G}{c^{2}} \psi$ which differs in sign from the equation above. This shows how Einstein's scalar equation of 1912 [4] is related to the time-time-component of the general relativistic tensor equation.

\section{Appendix}

In this appendix we wish to give a short direct proof that (4.2) satisfies $\mathbf{P}$, i.e., that $\delta A=c^{2} \delta M_{g}$.

Using the generally valid equation (2.3), now with $\phi$ replacing $\varphi$, we must eliminate $\rho$ via (3.3). This is most easily done if we set $\phi=c^{2} \psi^{2}$ and use (1.5). We obtain:

$$
\begin{aligned}
\delta A & =\frac{c^{4}}{2 \pi G} \int_{R^{3}} \psi^{2} \delta\left[\frac{\Delta \psi}{\psi}\right] d V=\frac{c^{4}}{2 \pi G} \int_{R^{3}}[\psi \Delta(\delta \psi)-(\Delta \psi) \delta \psi] d V \\
& =\frac{c^{4}}{2 \pi G} \int_{S_{\infty}^{2}} \vec{n} \cdot[\psi \vec{\nabla}(\delta \psi)-(\vec{\nabla} \psi) \delta \psi] d o .
\end{aligned}
$$


Now, the conditions for large $r$ imply that $\vec{\nabla} \psi$ falls off as fast as $1 / r^{2}$ and $\delta \psi$ as fast as $1 / r$. Hence the second term in the last bracket does not contribute. Therefore we may reverse its sign and obtain

$$
\delta A=\frac{c^{4}}{2 \pi G} \delta \int_{S_{\infty}^{2}}(\vec{n} \cdot \vec{\nabla} \psi) \psi d V=\frac{c^{2}}{4 \pi G} \delta \int_{S_{\infty}^{2}} \vec{n} \cdot \vec{\nabla} \phi d V=c^{2} \delta M_{g}
$$

\section{References}

[1] Geroch, R. (1978): "On the positive mass conjecture". In: Theoretical Principles in Astrophysics and Relativity, pp 245-252. Edited by N.R. Lebovitz, W.H. Reid, and P.O. Vandervort, University of Chicago Press.

[2] Visser, M. (1989): "A Classical Model For The Electron". Phys. Lett. A 139, 99-102.

[3] Robinson, T.R. (1995): "Mass and charge distributions of the classical electron". Phys. Lett. A 200, 335-339.

[4] Einstein, A. (1912): "Zur Theorie des statischen Gravitationsfeldes". Ann. Phys. (Leipzig) 38, 443-458. 